\title{
Overview of ALICE results in pp, pA and AA collisions
}

\author{
Rainer Schicker, for the ALICE Collaboration ${ }^{1, \star}$ \\ ${ }^{1}$ Phys. Inst., Im Neuenheimer Feld 226, 69120 Heidelberg, Germany
}

\begin{abstract}
The ALICE experiment at the Large Hadron Collider (LHC) at CERN is optimized for recording events in the very large particle multiplicity environment of heavyion collisions at LHC energies. The ALICE collaboration has taken data in $\mathrm{Pb}-\mathrm{Pb}$ collisions in Run I and Run II at nucleon-nucleon center-of-mass energies $\sqrt{s_{\mathrm{NN}}}=2.76$ and $5.02 \mathrm{TeV}$, respectively, and in pp collisions at center-of-mass energies $\sqrt{s}=0.9,2.76$, $5.02,7,8$ and $13 \mathrm{TeV}$. The asymmetric system $\mathrm{p}-\mathrm{Pb}$ was measured at a center-of-mass energy $\sqrt{s_{\mathrm{NN}}}=5.02 \mathrm{TeV}$. Selected physics results from the analysis of these data are presented, and an outline of the ALICE prospects for Run III is given.
\end{abstract}

\section{Introduction}

The ALICE experiment at the Large Hadron Collider (LHC) at CERN is a general purpose detector optimized for the measurement of high-energy $\mathrm{Pb}-\mathrm{Pb}$ collisions [1]. The analysis of such collisions allows to study a variety of issues which are pertinent to an improved understanding of Quantum Chromodynamics (QCD), the theory of the strong interaction. At high temperatures and energy densities of the QCD medium, lattice QCD calculations predict a phase transition to a state of deconfined quarks and gluons [2]. High-energy nuclear collisions allow to reach such energy densities, however within a finite volume and for a limited time only. This phase transition is accompanied by the restoration of chiral symmetry, in which the quarks acquire their current mass. The understanding of the properties of the QCD medium created in heavy-ion collisions necessitates a good knowledge of the underlying collision dynamics. Intrinsic QCD-medium signals can be disentangled from initial cold-matter and final-state effects by comparing the heavy-ion observables to the corresponding quantities measured in $\mathrm{pp}$ and $\mathrm{p}-\mathrm{Pb}$ collisions.

\section{The ALICE Experiment}

\subsection{The History of the ALICE Experiment}

The first conceptual ideas for experiments at the LHC were discussed in a workshop in 1990. Out of this workshop, an Expression of Interest, followed by a Letter of Intent and the Technical Proposal evolved. After approval of the ALICE central barrel system in 1997, the different detector systems were specified in Technical Design Reports. A muon spectrometer was later added, as well as a Transition Radiation Detector (TRD) and Electromagnetic Calorimeters EMCAL and DCAL in the central barrel.

^e-mail: schicker@physi.uni-heidelberg.de 


\subsection{The ALICE Physics Programme}

\subsubsection{The ALICE Physics Programme in pp-Collisions}

The ALICE physics programme in pp collisions is complementary to the other LHC experiments due to the low $\mathrm{p}_{T}$-threshold, and due to the excellent particle identification capability in the central barrel [3]. Besides being a reference for the analysis of $\mathrm{Pb}-\mathrm{Pb}$ collisions, the pp collisions have interest in their own. The analysis of minimum-bias pp collisions addresses a plethora of issues relevant for the understanding of soft QCD processes, such as particle and multi-particle production in the non-perturbative QCD regime. The analysis of high-multiplicity pp collisions allows to study the possible onset of collectivity in small systems, such as evidenced in collective flow effects.

\subsubsection{The ALICE Physics Programme in pA-Collisions}

The analysis of data taken in $\mathrm{p}-\mathrm{Pb}$ collisions, and its comparison to the corresponding quantity measured in $\mathrm{Pb}-\mathrm{Pb}$ collisions, can be used to disentangle cold-matter initial and final-state effects from intrinsic QCD-medium effects. Of particular interest here are possible shadowing of nuclear Parton Distribution Functions (nPDFs), and the low-x behaviour of the gluon-PDF as signature of gluon saturation at low values of Bjorken- $x$.

\subsubsection{The ALICE Physics Programme in AA-Collisions}

The analysis of $\mathrm{Pb}-\mathrm{Pb}$ collisions at LHC energies addresses a multitude of physics issues, above all the question of experimental observables to characterize the nature of the QCD phase diagram [4]. The observables derived in these analyses, and their correlations, can be used to study experimental signatures of the deconfinement and the chiral symmetry transition, and to examine the relationship between these two transitions.

\subsection{The ALICE Detector Systems}

In figure 1, the yoke of the ALICE magnet is shown in red. The innermost detector system, the Inner Tracking System (ITS), is zoomed in on the top right of the figure, showing the strip, drift and pixel subsystems. Radially outwards from the ITS, the Time Projection Chamber (TPC), the Transition Radiation Detector (TRD), and the Time-of-Flight detector (TOF) are shown. The V0 system serves as trigger detector, and its information is used to define event parameters such as high-multiplicity or rapidity-gap characteristics. In addition, there exist other small acceptance systems for particle identification and event characterization (FMD, HMPID, T0). The electromagnetic calorimeters EMCAL and PHOS are able to measure photons in a limited solid angle of the central barrel. A muon spectrometer complements the central barrel on one side within the pseudorapidity range $-4<\eta<-2.5$.

\subsection{The ALICE Performance}

The ALICE detector systems shown in figure 1 provide the information for reconstructing the individual tracks, for fitting their momentum, and for identifying the particle type of the track.

The ALICE particle identification capability by the energy loss $\mathrm{dE} / \mathrm{dx}$ in the TPC is shown on the left part, and by the Time-of-Flight as measured by the TOF detector on the right part of figure 2 . The information from the TRD detector, located between the TPC and the TOF detector, is used to identify electrons. The separation of electrons from charged hadrons can in addition be achieved by the electromagnetic calorimeters. 


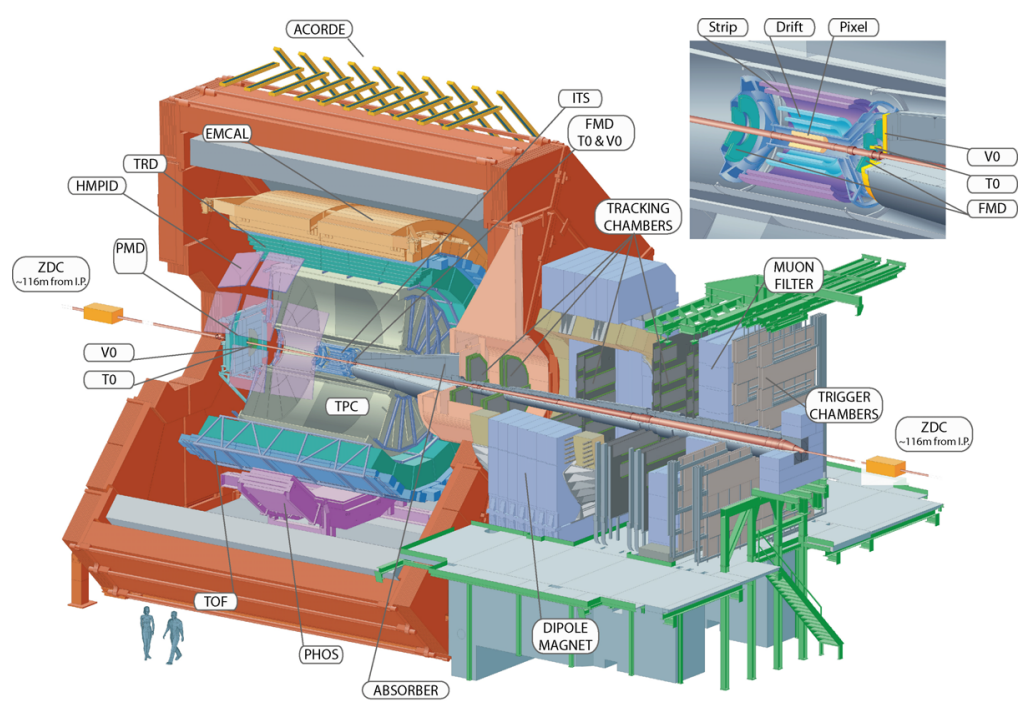

Figure 1: The detector systems of the ALICE central barrel, with the muon spectrometer shown on the right hand side of the central barrel.
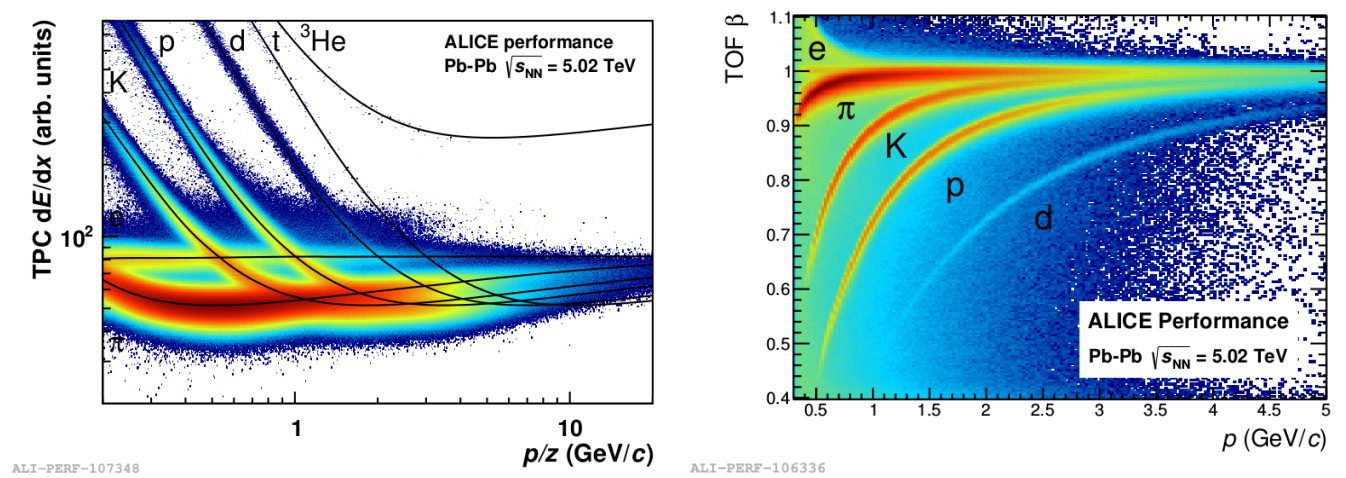

Figure 2: The particle identification by energy loss $\mathrm{dE} / \mathrm{dx}$ in the TPC is shown on the left. The particle identification by TOF is shown on the right.

\subsection{The Data Sample collected by ALICE}

The statistics of the data samples recorded by the ALICE collaboration in Run I and Run II of the LHC is displayed in table 1.

In Run I and Run II, the ALICE collaboration has taken data in pp collisions at center-of-mass energies of $\sqrt{s}=0.9,2.76,5.02,7,8$ and $13 \mathrm{TeV}$. In Pb-Pb collisions, events were recorded in Run I and Run II at center-of-mass energies of $\sqrt{s_{\mathrm{NN}}}=2.76$ and $5.02 \mathrm{TeV}$, respectively. In p-Pb collisions, data were recorded in Run I at the center-of-mass energy of $\sqrt{s_{\mathrm{NN}}}=5.02 \mathrm{TeV}$. During November and December of this year, data taking in $\mathrm{p}-\mathrm{Pb}$ collisions is planned at $\sqrt{s_{\mathrm{NN}}}=5.02$ and $8 \mathrm{TeV}$. 
Table 1: LHC Runs I+II, 2009-2015:

\begin{tabular}{lllll}
\hline system & run & year & $\sqrt{s_{\mathrm{NN}}}(\mathrm{TeV})$ & int. lumi. \\
\hline p-p & I & $2009-2010$ & 0.9 & $0.15 \mathrm{nb}^{-1}$ \\
p-p & I & 2011 & 2.76 & $1.1 \mathrm{nb}^{-1}$ \\
p-p & I & $2010-2011$ & 7 & $4.8 \mathrm{pb}^{-1}$ \\
p-p & I & 2012 & 8 & $9.7 \mathrm{pb}^{-1}$ \\
p-p & II & 2015 & 5.02 & $2.5 \mathrm{nb}^{-1}$ \\
p-p & II & 2015 & 13 & $4.35 \mathrm{pb}^{-1}$ \\
p-Pb & I & 2013 & 5.02 & $30 \mathrm{nb}^{-1}$ \\
$\mathrm{~Pb}-\mathrm{Pb}$ & I & 2010,2011 & 2.76 & $0.1 \mathrm{nb}^{-1}$ \\
$\mathrm{~Pb}-\mathrm{Pb}$ & II & 2015 & 5.02 & $0.24 \mathrm{nb}^{-1}$ \\
\hline
\end{tabular}

\section{Proton-Proton Collisions}

\subsection{Multi-Parton Interactions}

Particle production in high-energy hadronic collisions can get substantial contributions from MultiParton Interactions (MPI). Here, several partonic interactions can take place in a hadronic collision, resulting in a multiplicity dependence of observables such as particle yields and transverse momentum distributions. Analyzing MPI effects is of fundamental importance for studying the range of validity of Parton Distribution Functions (PDFs), an input when calculating perturbative QCD cross sections.
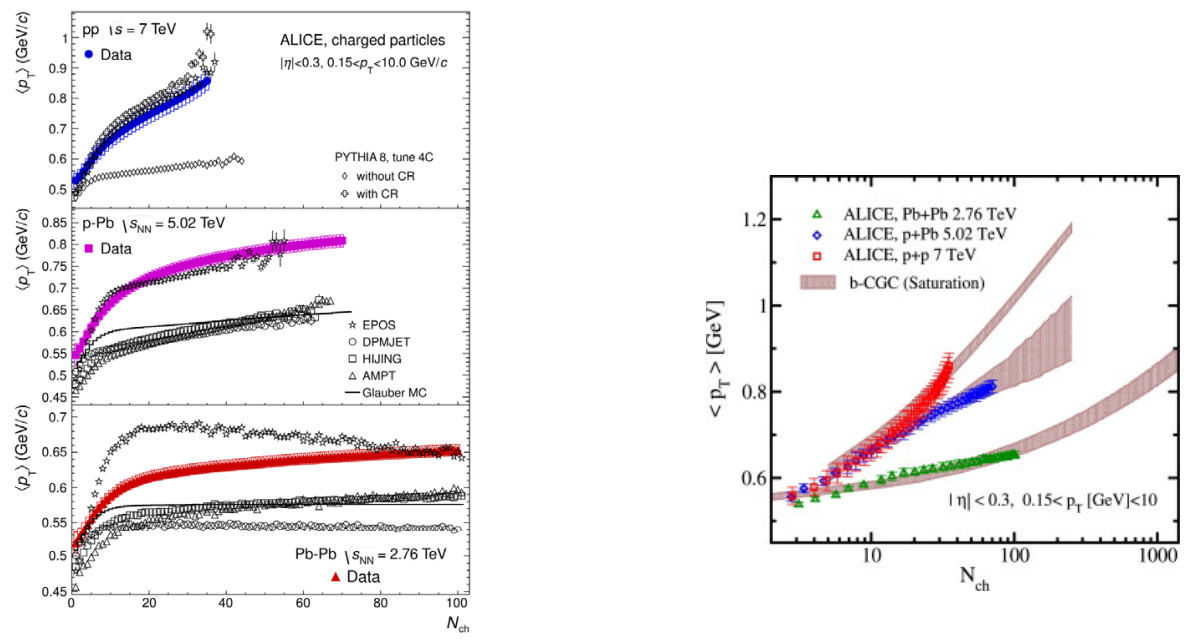

Figure 3: Average transverse momentum $\left\langle p_{T}>\right.$ as function of charged particle multiplicity for pp, $\mathrm{p}-\mathrm{Pb}$ and $\mathrm{Pb}-\mathrm{Pb}$ collisions are shown on the left. The comparison of measured $\left\langle p_{T}>\right.$ to the predictions of the Color Glass Condensate approach are shown on the right.

In the top left panel in figure 3, the ALICE measurement of the average transverse momentum in the range $|\eta|<0.3$ for pp collisions at $\sqrt{s}=7 \mathrm{TeV}$ is displayed as function of the charged particle multiplicity [5]. The results of the PYTHIA 8 calculations, shown by the open symbols, clearly show 
the importance of including colour reconnections. In the middle panel on the left, the corresponding ALICE data for $\mathrm{p}-\mathrm{Pb}$ collisions are shown in purple, superimposed in black by the predictions of the EPOS, DPMJET, HIJING and AMPT models [6]. The EPOS model predicts the ALICE data reasonably well, even though it shows a different trend at multiplicities $\mathrm{N}_{c h}<20$ [7]. None of the other three models is able to describe the data. A Glauber MC calculation, shown by the solid black line, also fails to reproduce the data. In this Glauber $\mathrm{MC}$ approach, $\mathrm{p}-\mathrm{Pb}$ collisions are assumed to consist of independent pp collisions, each contributing to the particle yield according to the yield as measured in pp collisions. In the bottom panel on the left, the ALICE data for $\mathrm{Pb}-\mathrm{Pb}$ collisions are shown in red, superimposed in black by the predictions of the different model calculations. As for the p-Pb data, the DPMJET, HIJING and AMPT model as well as the MC Glauber calculation underpredict the measured $\left\langle p_{T}\right\rangle$. The EPOS model overpredicts the data and shows a different trend.

On the right side of figure 3, the ALICE data are compared to Color Glass Condensate (CGC) model calculations [8]. The CGC approach, addressing the importance of the initial-state effect, is based on gluon saturation at low values of Bjorken- $x$, and Glasma physics. The CGC predictions are in much better agreement with the ALICE data than the model predictions shown on the left of figure 3 .

\subsection{Strangeness and Charm in Proton-Proton Collisions}

The initial state of proton-proton collisions does not contain strange valence quarks. The measurement of strange hadrons hence allows the study of particle production in QCD both in the perturbative and non-perturbative sector. Strangeness can be created in hard perturbative $2 \rightarrow 2$ partonic scatterings by flavour creation $(g g \rightarrow s \bar{s}, q \bar{q} \rightarrow s \bar{s})$ and by flavour excitation $(g s \rightarrow g s, q s \rightarrow q s)$. During partonic evolution, gluon splitting $(g \rightarrow s \bar{s})$ contributes to strangeness production. At low transverse momenta, strangeness production is dominated by non-perturbative processes such as string fragmentation.

On the left of figure 4 , the $\mathrm{p}_{T}$-integrated yield ratios of strange $\left(K_{S}^{0}, \Lambda, \bar{\Lambda}\right)$ and multi-strange $\left(\Xi^{-}, \overline{\Xi^{+}}, \Omega, \overline{\Omega^{+}}\right.$) hadrons measured in pp, p-Pb and $\mathrm{Pb}-\mathrm{Pb}$ are shown [9]. These ratios represent a production rate which increases faster for strange hadrons than for non-strange hadrons. The strangeness enhancement increases with hadron strangeness, and with event activity defined by $\mathrm{dN}_{c h} / \mathrm{d} \eta$. This enhancement is not reflected by the PYTHIA generator shown in figure 4 by the solid lines. The EPOS generator describes the trend vs multiplicity, even though it fails for the triply strange $\Omega$-Baryon. The Dipsy model describes the data best, but is also not doing well for the triply $\Omega$-Baryon.

On the top right part of figure 4 , the yield $\mathrm{dN}_{J / \Psi} / \mathrm{dy}$, normalized to $<\mathrm{dN}_{J / \Psi} / \mathrm{dy}>$, is shown as function of the normalized charged particle multiplicity $\left(\mathrm{dN}_{c h} / \mathrm{d} \eta\right) /<\mathrm{dN}_{c h} / \mathrm{d} \eta>$. This yield is shown in red at midrapidity $|\mathrm{y}|<0.9$, and in blue at forward rapidities $2.5<|\mathrm{y}|<4$.0. An approximate linear correlation of these quantities is seen in figure 4 . In the bottom right part, the normalized $\mathrm{dN}_{J / \Psi} / \mathrm{dy}$ yield as predicted by PYTHIA 6.4 in Perugia 2011 tune is shown. In this calculation, only J/ $\Psi$ 's produced in hard scatterings via the NRQCD framework are calculated, whereas $\mathrm{J} / \Psi$ production from cluster formation during parton shower evolution or in MPI are not taken into account.

\subsection{Charmed Mesons in Proton-Proton Collisions}

The study of open-charm meson production in pp collisions at LHC energies allows to test predictions of perturbative QCD (pQCD) at the highest collider energies available. Such pQCD calculations are done in collinear factorization approach at next-to-leading order in the general-mass variable-flavour-number scheme (GM-VFNS), or at fixed order with next-to-leading-log resummation (FONLL). These calculations describe the $\mathrm{p}_{T}$-differential production cross sections of D-mesons within uncertainties. 


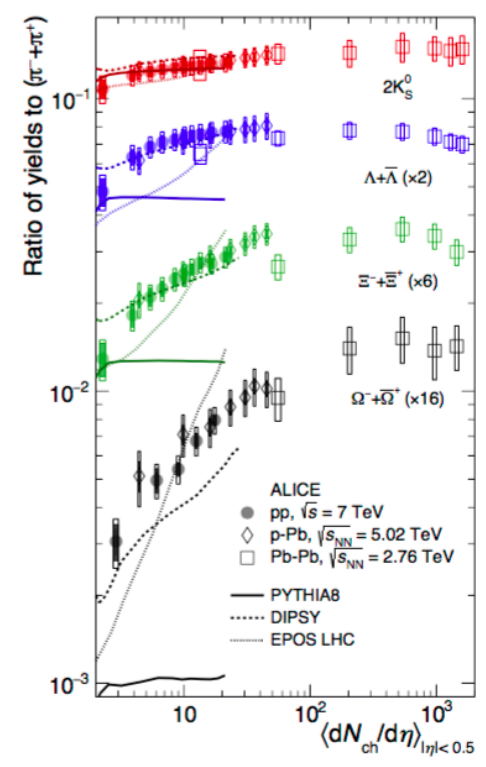

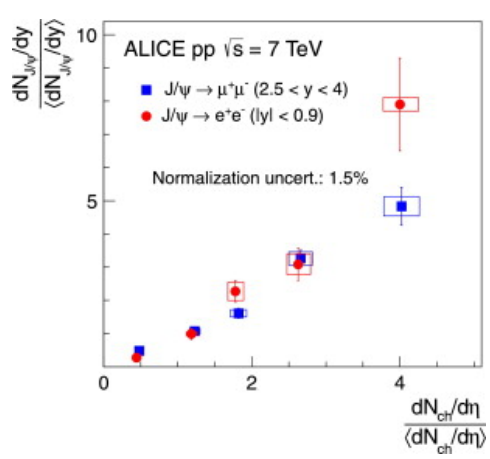

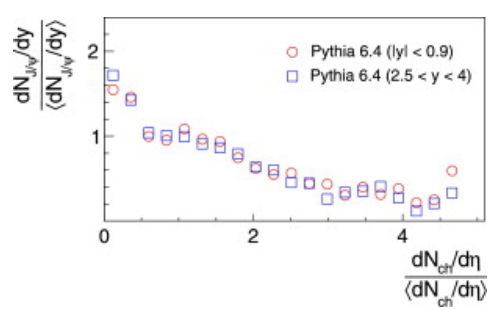

Figure 4: The yield ratio of strange and multi-strange hadrons to pions as function of $(\mathrm{dN} / \mathrm{d} \eta)$ is shown on the left. The normalized $\mathrm{J} / \Psi$ yield as function of normalized $\mathrm{dN}_{c h} / \mathrm{d} \eta$ is shown in the upper part on the right. The PYTHIA prediction of this normalized yield is shown in the lower part on the right.

The relative D-meson yield (average of $\mathrm{D}^{0}, \mathrm{D}^{+}, \mathrm{D}^{*+}$ ) is shown in figure 5 in four $\mathrm{p}_{T}$-intervals as function of the relative charged particle yield [10]. These measurements are compared to model predictions by PYTHIA 8 (red-dotted line), the EPOS models (green-dashed and green dot-dashed line), and the percolation model (blue dot-dashed line). In PYTHIA 8, heavy-flavour production is due to four mechanisms, the first hard $2 \rightarrow 2$ hard process, the subsequent hard process in MPI, gluon splitting from hard process, and from initial/final state gluon radiation (ISR/FSR). The dominating contribution in PYTHIA 8 at LHC energies is the ISR/FSR mechanism which contributes $62 \%$ and $40 \%$ for D and B-meson production, respectively. The EPOS model incorporates initial conditions according to the Gribov-Regge multiple scattering framework. Individual scatterings are initiated by Pomerons, and are associated to parton ladders. Each of these parton ladders represents a hard pQCD process with initial and final state radiation. A saturation scale is introduced for taking account of nonlinear effects. With these initial conditions, a hydrodynamical evolution can be defined on the core of the collision. The EPOS calculation without hydro-evolution predicts an approximate linear increase of D-meson production as function of relative charged-particle multiplicity. The EPOS model with hydro-evolution results in a deviation from linear dependence. Within the percolation model, highenergy hadronic collisions are dominated by the exchange of colour sources of finite spatial dimension between projectile and target [11]. The percolation model predicts a faster than linear increase of the relative D-meson yield.

\section{Proton-Lead Collisions}

The analysis of the $\mathrm{p}-\mathrm{Pb}$ collision system sheds light on the role of initial and final-state effects when studying differences between the $\mathrm{pp}$ and $\mathrm{Pb}-\mathrm{Pb}$ systems [12]. The nuclear shadowing of PDFs, the 


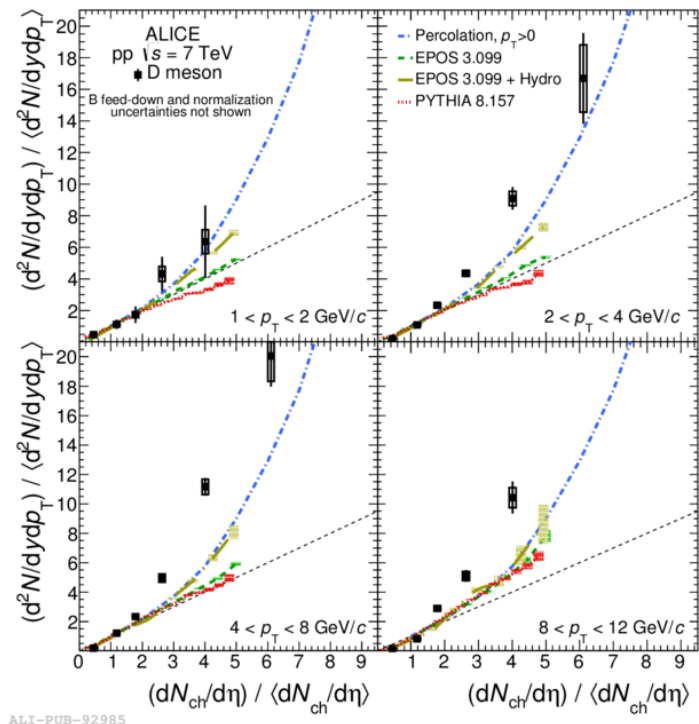

Figure 5: Average D-meson relative yield as function of relative charged-particle multiplicity at central rapidity in different $\mathrm{p}_{T}$-intervals.

existence of CGC in the initial state and the hydrodynamic phase in the evolution of the system, for example, affect bulk observables quite differently in the $\mathrm{pp}, \mathrm{p}-\mathrm{Pb}$ and $\mathrm{Pb}-\mathrm{Pb}$ collision systems.
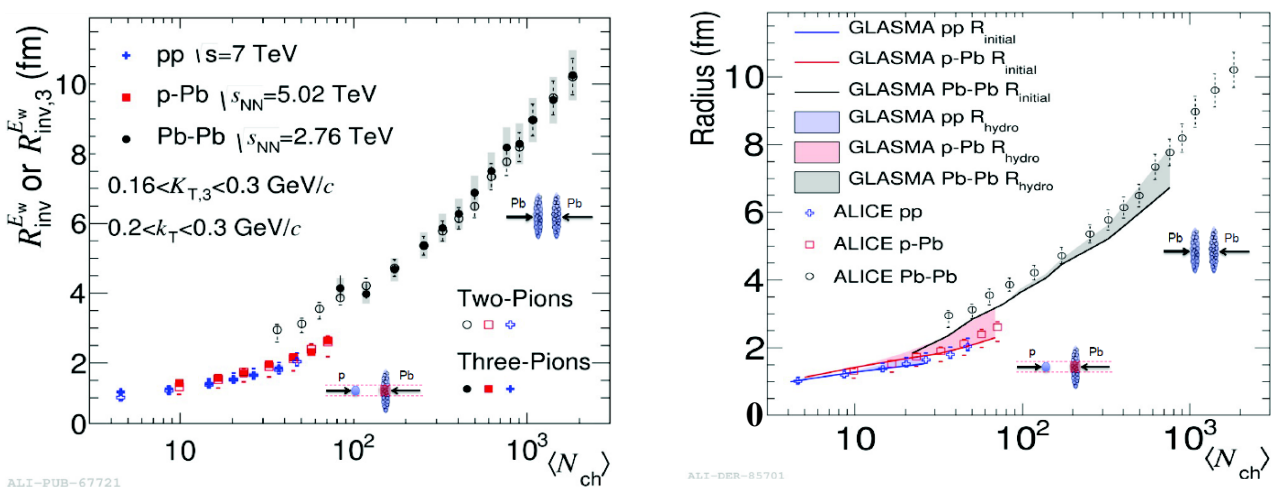

Figure 6: Pion source sizes are shown on the left, model calculations on the right.

The source sizes derived from pion correlations are shown on the left in figure 6 as a function of the charged particle multiplicity for the $\mathrm{pp}, \mathrm{p}-\mathrm{Pb}$ and $\mathrm{Pb}-\mathrm{Pb}$ systems [13]. Here, $R_{\text {inv }}$ expresses the invariant source radius derived by a Gaussian fit to the two-pion correlation function. The quantity $R_{i n v, 3}$ denotes the corresponding quantity as determined by analysis of three-pion cumulant distributions. At the same multiplicities within the range $60<N_{c h}<80$, the source radii in the pp and p-Pb systems 
show similar values, with a significantly larger value in the $\mathrm{Pb}-\mathrm{Pb}$ system reflecting the hydrodynamic expansion stage of a heavy-ion collision. These ALICE data are compared on the right of figure 6 to predictions by the CGC based IP-Glasma model [14].

\section{Lead-Lead Collisions}

\subsection{Exploring the QCD Phase Diagram with Heavy-Ion Collisions}

Collisions of heavy-ions at high energies produce QCD matter at extreme values of energy densities and temperatures [15]. Lattice gauge calculations indicate that a QCD phase transition takes place under such conditions. In lattice QCD approach, the partition function of the grand canonical ensemble is evaluated stochastically by Monte Carlo sampling of field configurations. Thermodynamic state functions such as pressure and energy densities can subsequently be evaluated. The transition from hadronic matter to a state of deconfined quarks and gluons, the Quark-Gluon Plasma (QGP) is seen in such calculations at a critical temperature of $\mathrm{T}_{\mathrm{cr}} \sim 160 \mathrm{MeV}$. At this critical temperature, the chiral condensate $\langle\bar{q} q\rangle$ is rapidly diminishing, thereby indicating the restoration of broken chiral symmetry [16]. A multitude of experimental observables are analyzed to characterize the nature of the QCD phase diagram, and to establish signals of partial chiral symmetry restoration. The present understanding of this deconfined state is a strongly coupled QGP which behaves as a liquid with a very low viscosity to entropy ratio [17].

\subsection{Soft Probes in Heavy-Ion Collisions}

The analysis of the azimuthal anisotropy of particle production in heavy-ion collisions reveals information on the initial geometry of the overlap zone of the colliding nuclei, on the equation of state of the produced QCD medium and its transport properties.
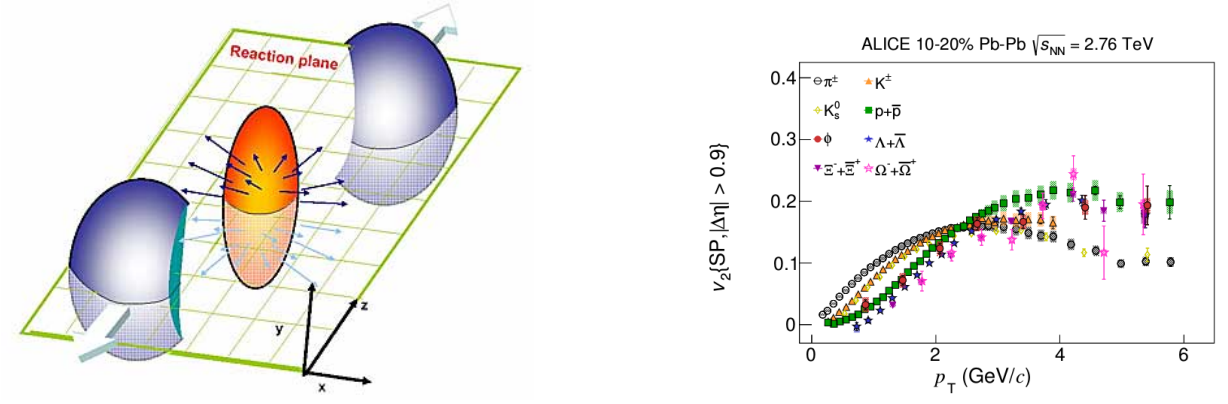

Figure 7: Heavy-ion collision geometry is shown on the left, $v_{2}$-flow coefficients on the right.

On the left side of figure 7, a heavy-ion collision geometry is shown. The azimuthal distribution of particles produced in the almond-shape overlap zone is analyzed with respect to the reaction plane by

$$
\frac{d^{2} N}{p_{T} d p_{T} d \phi} \propto 1+2 \sum_{n=1}^{\infty} v_{n}\left(p_{T}\right) \cos \left[n\left(\phi-\Psi_{n}\right)\right]
$$


The azimuthal particle distribution of 1 is characterized by the symmetry planes $\Psi_{n}$ and by the coefficients $v_{n}$. Such distributions test hydrodynamical models, and allow to extract properties of the QCD medium such as viscosity.

On the right side of figure 7 , the $v_{2}$-coefficient of the Fourier decomposition of the azimuthal anisotropy is shown for the centrality class $10 \%-20 \%$ [18]. A distinctive mass ordering of the flow coefficient $v_{2}$ is seen at low values of transverse momentum $\mathrm{p}_{T}$. This mass ordering is attributed to the interplay between elliptic and radial flow. Radial flow tends to deplete the spectrum at low values of $\mathrm{p}_{T}$, which increases with increasing particle mass and transverse velocity. In a system with azimuthal anisotropy, this depletion is larger in-plane than out-of-plane, thereby reducing $v_{2}$. At large values of $\mathrm{p}_{T}$, the flow coefficients $v_{2}$ show a tendency to order according to the particle type of baryon and meson. Such scaling was first observed at RHIC, and was interpreted as evidence that quark degrees of freedom dominate in the early stages of heavy-ion collisions when collective flow develops [19].

\subsection{Hard Probes in Heavy-Ion Collisions}

The analysis of jets produced in scatterings of highly virtual quarks or gluons provides an important tool to test the Standard Model in the perturbative region of QCD. In heavy-ion collisions, the analysis of jet quenchinq allows to study the QCD medium produced in the collision. In figure 8 on the left, a hard parton scattering is shown. The hadronization of the two partons after scattering results in two jets emerging on opposite sides in azimuthal angle. Of particular interest are hadron-photon and jet-photon correlations. The photon is expected to escape the interaction zone with much reduced interaction as compared to hadrons, and hence carries the information of the hard-scattering kinematics.

On the right of figure 8 , a dijet event is shown with the leading jet of transverse momentum $\mathrm{p}_{T}=205.1 \mathrm{GeV} / \mathrm{c}$, and the subleading jet of $\mathrm{p}_{T}=70.0 \mathrm{GeV} / \mathrm{c}$ at the opposite azimuthal angle [20].
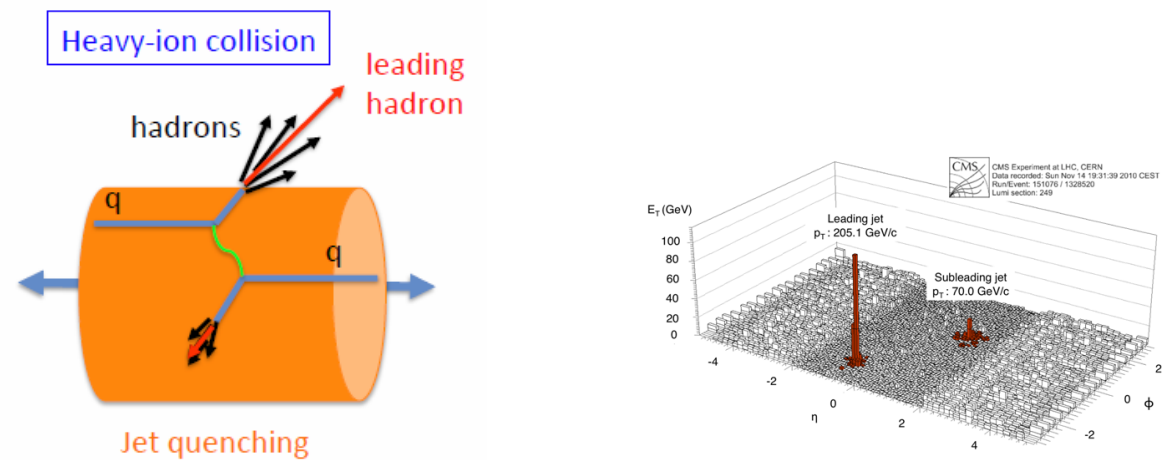

Figure 8: Hard parton scattering is shown on the left, a dijet event on the right (figure from [20]).

The energy of the partons of the jet is reduced in heavy-ion collisions as compared to pp collisions due to medium-induced gluon radiation and collisional energy loss. In perturbative QCD, the production cross section of the initial hard scattered parton can be calculated. The hadronization of the initial scattered parton can be calibrated by jet measurements in pp collisions. Jet quenching in heavy-ion collisions can be analyzed by studying whether these jet spectra can be understood as an incoherent superposition of nucleon-nucleon collisions. The analysis of ALICE data reveals that jets with transverse momenta $40<p_{T, j e t}<120 \mathrm{GeV} / \mathrm{c}$ in heavy-ion collisions are strongly suppressed in the $10 \%$ most central events [21]. 


\subsection{Low Mass Dileptons}

In the limit of vanishing quark masses, the QCD Lagrangian contains a symmetry due to the conserved right or left-handedness of the zero-mass spin-1/2 particles. This chiral symmetry is spontaneously broken, leading to a population of the QCD ground state by scalar quark-antiquark pairs [22]. In the QCD medium formed in heavy-ion collisions at high energies, the expected partial restoration of chiral symmetry is reflected by significant modifications of hadron properties. Of particular importance for the study of such in-medium modifications is the $\rho$-meson. Due to the short life-time of $c \tau=1.3 \mathrm{fm}$, $\rho$-decays occur predominantly within the QCD-medium formed in the heavy-ion collision. The measurement of the in-medium $\rho$-spectral function through the $\rho$-dilepton decays allows to test the nature of the formed QCD medium. Both an in-medium mass shift, as well as a broadening of the $\rho$-meson have been advocated [23, 24].
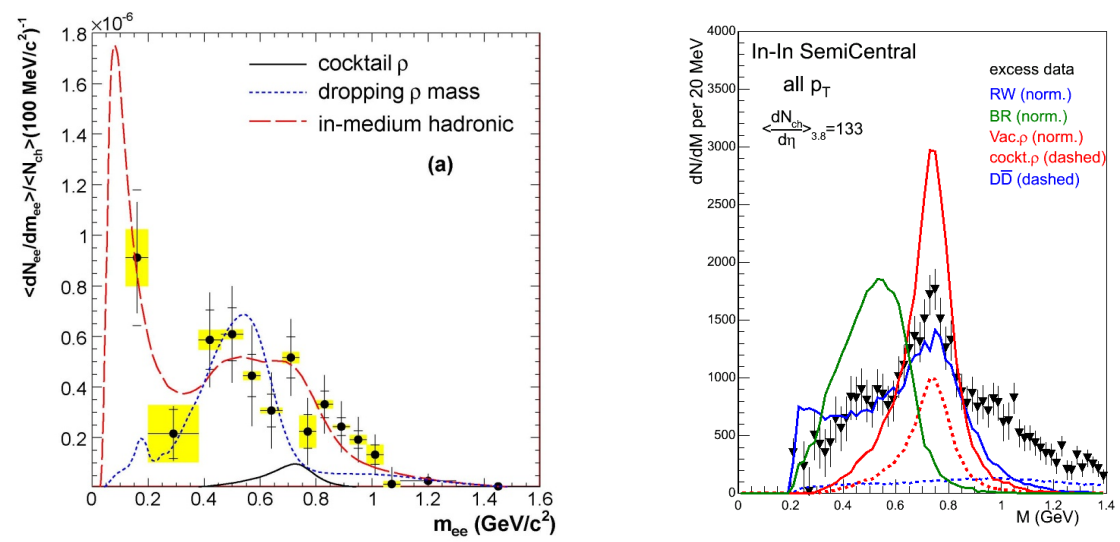

Figure 9: Invariant dilepton mass spectra measured by CERES (left) and NA60 (right).

In figure 9 on the left, the invariant mass distribution of $e^{+} e^{-}$-pairs measured by the CERES collaboration in $\mathrm{Pb}-\mathrm{Au}$ collisions at $158 \mathrm{~A} \mathrm{GeV} / \mathrm{c}$ is shown [25]. The data points represent the $e^{+} e^{-}-$ pair yield after subtraction of the hadronic cocktail, i.e. the contribution to this yield from known sources such as $\pi^{0}, \eta, \eta^{\prime}$ and $\omega$ Dalitz-decays. In this figure, the dotted and long-dashed lines represent the expectations as derived from the dropping and broadening $\rho$-mass scenario, respectively. Within the statistics of these data, the dropping $\rho$-mass scenario is disfavored. On the right of figure 9 , the excess of the $\mu^{+} \mu^{-}$-pair yield measured by the NA60 collaboration in In-In collisions is shown [26]. These data are compared to expectations from the dropping $\rho$-mass scenario (green line) and the broadening $\rho$-mass scenario (blue line). The solid red line represents the expectation of this yield in case of no changes to the $\rho$-spectral function. The dashed red line is shown for illustration, and indicates the expected contribution from the cocktail $\rho$ bound by the ratio of $\rho / \omega=1.2$ measured at high $\mathrm{p}_{T}>1.6 \mathrm{GeV} / \mathrm{c}$. $>$ From these data, an unmodified $\rho$ is clearly ruled out, as well as the dropping mass scenario. The most realistic description of these data is within the broadening $\rho$-mass scenario.

The ALICE collaboration has measured the yield of low-mass dileptons at pseudorapidities $|\eta|<0.8$ in Run I of the LHC in pp collisions at $\sqrt{s}=7 \mathrm{TeV}$, and in p-Pb collisions at $\sqrt{s_{\mathrm{NN}}}=5.02 \mathrm{TeV}$.

The differential cross section for $e^{+} e^{-}$-pair production in pp collisions is shown in figure 10 on the left. Superimposed to the data are the cocktail contributions expected from $\pi^{0}, \eta, \eta^{\prime}, \omega, \phi$ and $J / \Psi$ Dalitz-decays, as well as from the two-body $e^{+} e^{-}$-decays of $\rho, \omega, \phi$ and $J / \Psi$. At pair masses 

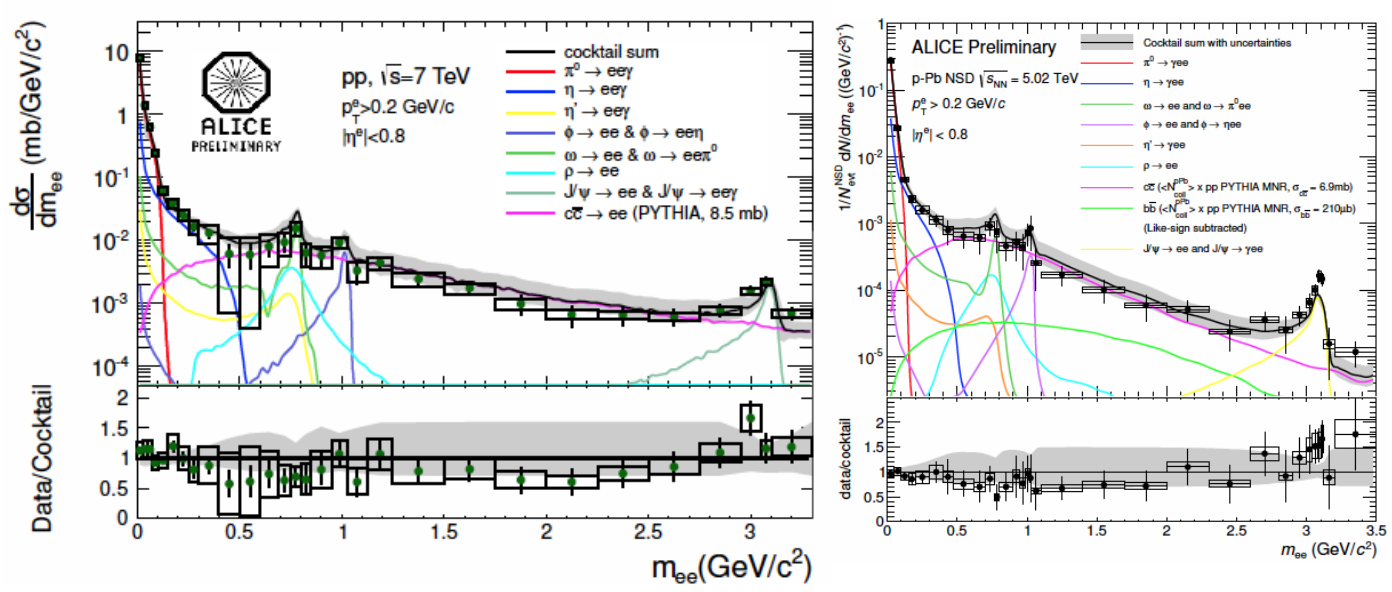

Figure 10: ALICE mass spectra of $e^{+} e^{-}$-pairs in pp (left) and p-Pb collisions (right).

beyond the $\phi$-mass, the spectrum is dominated by the contribution of $c \bar{c}$ decays. The expected cocktail contribution is consistent with the data up to a mass of $m=3.5 \mathrm{GeV} / \mathrm{c}^{2}$ as shown in the lower part on the left. The differential cross section for $e^{+} e^{-}$-pair production in $\mathrm{p}-\mathrm{Pb}$ collisions is shown in figure 10 on the right, together with the expected cocktail contributions. The expected cocktail contribution is consistent with the data up to a mass of $\mathrm{m}=3.5 \mathrm{GeV} / \mathrm{c}^{2}$ as shown in the lower part on the right.

The dilepton spectra measured in $\mathrm{pp}$ and $\mathrm{p}-\mathrm{Pb}$ collisions in ALICE serve as baseline to the corresponding measurements in $\mathrm{Pb}-\mathrm{Pb}$ collisions. Besides the in-medium modification of low-mass vector mesons discussed above, the analysis of virtual direct photons in the low mass region and signatures of thermal radiation from the QGP in the intermediate mass region are of high interest. Such studies can be pursued in ALICE by analysing dielectrons in the central barrel as well as by examining dimuons in the ALICE muon spectrometer.

\section{The Upgrade Programme of ALICE}

The ALICE detector upgrade programme is driven by the future ALICE physics plans [27]. These plans center on rare physics probes where the ALICE capabilities on measuring low- $\mathrm{p}_{T}$ tracks, in conjunction with excellent particle identification, are unique. Such probes are, for example, heavy flavour hadrons and quarkonia at low $\mathrm{p}_{T}$, low mass lepton pairs, jets, light nuclei and hypernuclei. These upgrade plans are formulated under the assumption that the LHC will increase the Pb-beam intensity such that an interaction rate of $50 \mathrm{kHz}$ is reached in Run III, corresponding to an instantaneous luminosity of $\mathrm{L}=6 \times 10^{27} \mathrm{~cm}^{-2} \mathrm{~s}^{-1}$. Standard trigger strategies cannot be applied in most cases of the above-mentioned rare probes. The ALICE detectors hence need to be modified such that all interactions can be inspected. Based on these considerations, the ALICE detector upgrade programme for Run III starting in the year 2021 consists of

- Upgrade of the TPC in order to operate in ungated mode to have a dead-time free readout

- A new high-resolution, thin-material Inner Tracking System (ITS). The resolution on distance of closest approach will be improved by a factor of about 3 as compared to the present performance

- Upgrade of the readout electronics of the TRD, TOF, PHOS detectors and the muon spectrometer for the future high-rate data taking 
- Upgrade of the data acquisition and the high-level trigger system for the future high-rate data taking

- Upgrade of the offline processing software in order to handle the much larger number of events

\section{Summary and Outlook}

The ALICE collaboration has taken data in Run I and Run II of the LHC in pp, p- $\mathrm{Pb}$ and Pb-Pb collisions. A multitude of physics analyses of the $\mathrm{Pb}-\mathrm{Pb}$ data addresses the nature of the QCD phase diagram. The study of $\mathrm{pp}$ collisions addresses a variety of questions relevant for an improved understanding of the non-perturbative sector of QCD. The analyses of $\mathrm{p}-\mathrm{Pb}$ collisions reveal effects of cold-matter initial and final-state effects in heavy-ion induced reactions. The future of the ALICE physics programme is centered on measuring rare probes with much improved statistics as is presently available. The ALICE detectors are correspondingly upgraded in order to meet the increased data rate in the future.

\section{Acknowledgements}

This work is supported by the German Federal Ministry of Education and Research under promotional reference 05P15VHCA1.

\section{References}

[1] ALICE Collaboration (F. Carminati et al.), J. Phys. G 30, 1517 (2004).

ALICE Collaboration (B. Alessandro et al.), J. Phys. G 32, 1295 (2006)

[2] HotQCD Collaboration (A. Bazavov et al.), Phys. Rev. D 90, 094503 (2014)

[3] ALICE Collaboration (B. Abelev et al.), Int. J. Mod. Phys. A 29, 1430044 (2014)

[4] P. Braun-Munzinger and J. Wambach, Rev. Mod. Phys. 81, 1031 (2009)

[5] ALICE Collaboration (B. Abelev et al.), Phys. Lett. B 727, 371 (2013)

[6] S. Roesler, R. Engel and J. Ranft, DPMJET-III, (2000), arXiv:hep-ph/0012252.

X.-N. Wang and M. Gyulassy, Phys. Rev. D 44, 3501 (1991).

Z.-W. Liu, C.M. Ko, B.-A. Li, B. Zhang and S. Pal, Phys. Rev. C 72, 064901 (2005)

[7] T. Pierog, I. Karpenko, J. Katzy, E. Yatsenko and K. Werner, arXiv:1306.0121

[8] A.H. Rezaeian, Phys. Lett. B 727, 218 (2013)

[9] ALICE Collaboration (J. Adam et al.), arXiv:1606.07424

[10] ALICE Collaboration (J. Adam et al.), JHEP 1509, 148 (2015)

[11] E.G. Ferreiro and C. Pajares, arXiv:1501.03381

[12] ALICE Collaboration (J. Adam et al.), Phys. Rev. C 91(6), 064905 (2015)

[13] ALICE Collaboration (B. Abelev et al.), Phys. Lett. B 739, 139 (2014)

[14] B. Schenke and R. Venugopalan, Phys. Rev. Lett. 113, 102301 (2014)

[15] Y. Akiba et al., arXiv:1502.02730

[16] W. Weise, Nucl. Phys. A 690, 98 (2001)

[17] H. Niemi, K.J. Eskola, R. Paatelainen and K. Tuominen, Nucl. Phys. A 956, 312 (2016)

[18] ALICE Collaboration (B. Abelev et al.), JHEP 1506, 190 (2015)

[19] STAR Collaboration (B.I. Abelev et al.), Phys. Rev. C 75, 054906 (2007).

PHENIX Collaboration (A. Adare et al.,), Phys. Rev. Lett. 98, 162301 (2007)

[20] CMS Collaboration (S. Chatrchyan et al.), Phys. Rev. C 84, 024906 (2011) 
[21] ALICE Collaboration (J. Adam et al.), Phys. Lett. B 746, 1 (2015)

[22] A.W. Thomas and W. Weise, The Structure of the Nucleon (Wiley-VCH, Berlin, 2001) 149

[23] G.E. Brown and M. Rho, Phys. Rept. 363, 85 (2002)

[24] R. Rapp and J. Wambach, Adv. Nucl. Phys. 25, 1 (2000)

[25] CERES Collaboration (D. Adamova et al.), Phys. Lett. B 666, 425 (2008)

[26] NA60 Collaboration (R. Arnaldi et al.), Phys. Rev. Lett. 96, 162302 (2006)

[27] ALICE Collaboration (B. Abelev et al.), J. Phys. G 41, 087001 (2014) 